\title{
Asthma phenotypes, comorbidities and disease activity in COVID-19: the need of risk stratification Reply to Morais-Almeida
}

\author{
Giulia Carli ${ }^{1}$, Lorenzo Cecchi ${ }^{2}$, Justin Stebbing ${ }^{3}$, Paola Parronchi ${ }^{4}$, and Alessandro Farsi ${ }^{1}$ \\ ${ }^{1}$ Azienda USL Toscana centro \\ ${ }^{2}$ Azienda USL Toscana centro Sedi di Prato \\ ${ }^{3}$ Imperial College Healthcare NHS Trust \\ ${ }^{4}$ University of Florence
}

June 23, 2020

\section{Reply to Morais-Almeida.}

To the Editor,

We appreciate Dr. Morais-Almeida's comments ${ }^{1}$ about our Letter to the Editor, presenting additional literature about asthma prevalence in severe COVID-19 patients and highlighting data that contrasts our hypothesis that asthma, particularly type 2 asthma, may be protective against severe disease.

The data that protection may be dependent on type 2 immunity is derived from the higher percentage of asthmatics being atopic ${ }^{2}$, also reflected in the series of $\sim 2,500$ patients regularly followed up in our Allergy Unit. $\mathrm{Yu}$ et al. ${ }^{3}$ provided preliminary evidence about this in a single-center retrospective study, where COVID-19 atopic patients had less severe infections, milder lung damage compared to age- and gendermatched COVID-19 controls.

ACE-2, the SARS-CoV-2 receptor, is linked to type 1 and 2 interferon signatures, and found to be overexpressed in type 2-low asthmatics ${ }^{4}$. Nevertheless, different outcomes in distinct asthma phenotypes still need to be addressed in COVID-19 studies.

Besides Italy and China, reports from Russia ${ }^{5}$ on $~ 1,300$ intensive care unit patients with SARS-CoV-2 infection confirm the observation of a low prevalence of chronic lung diseases (i.e. asthma as well as COPD).

Although preliminary data on the first COVID-19 cases in the US 6 seem to contrast these observations, the higher prevalence of asthma in US COVID-19 hospitalised patients should be considered alongside a higher overall prevalence in these countries compared to Europe and China, as well as on the influence of other comorbidities (i.e. obesity) and host factors (i.e. age, race: $33 \%$ were non-Hispanic black patients in the study by Garg et al.) impacting COVID-19 outcomes. Another report from Sweden ${ }^{7}$ highlights the association between severe asthma and severe COVID-19.

The severe asthma phenotype is often characterized by mixed granulocytic populations (neutrophilic and eosinophilic), prevalent type 1 inflammation, increased IFN- $\gamma$ levels in the airways and ineffectiveness of ICS. This severe phenotype by itself, although accounting for less than $5 \%$ of asthmatic patients, would justify the CDC (and other institutions) including asthma as a risk factor for COVID-19. Data from the UK ${ }^{8}$, apart from confirming the role of additional comorbidities, draw attention to the recent use of oral steroids, which, indeed, may be a clue for uncontrolled and/or severe asthma.

Uncontrolled asthma is a risk factor for viral exacerbations and hospitalizations and we embrace the opportunity to stress the importance of optimal adherence to asthma controlling medications, regular follow-up 
and specialist-assessment of disease activity. Moreover, treatable comorbidities, which may impair asthma control, should always be managed. Promoting vaccination for preventable respiratory infections (i.e. Influenza and Pneumococcal pneumonia) is also advisable. Future studies may help better distinguishing the impact of different asthma phenotypes and comorbidities on COVID-19 outcome.

Carli G. ${ }^{1}$, Cecchi L. ${ }^{1}$, Stebbing J. ${ }^{2}$, Parronchi P. ${ }^{3}$, Farsi A. ${ }^{1}$

${ }^{1}$ SOS Allergy and Clinical Immunology, USL Toscana Centro, Prato Italy

${ }^{2}$ Department of Surgery and Cancer, Imperial College London, London SW7 2AZ, UK

${ }^{3}$ Department of Experimental and Clinical Medicine, University of Florence, Florence, Italy

\section{Conflicts of interests}

Dr. Carli, Dr. Cecchi, Prof. Parronchi, and Dr. Farsi have nothing to disclose. Prof. Stebbing's conflicts of interest can be found athttps://www.nature.com/onc/editorsand none are relevant here.

\section{References}

1. Morais-Almeida M, Barbosa M, Sousa CS, Aguiar R, Bousquet J, Update on asthma prevalence in severe COVID-19 patients, Allergy. press

2. Papi A, Brightling C, Pedersen SE, Reddel HK. Asthma. Lancet. 2018 Feb 24;391(10122):783-800. doi: 10.1016/S0140-6736(17)33311-1

3. Wendan S, Ziang G, Yongjun D, Ting Z, Wei Z, Yu X, Clinical characteristics of COVID-19 patients combined with allergy, Allergy. 2020. Doi: 10.1111/ALL.14434

4. Camiolo MJ, Gauthier M, Kaminski N, Ray A, Wenzel SE. Expression of SARS-CoV-2 Receptor ACE2 and Coincident Host Response Signature Varies by Asthma Inflammatory Phenotype [published online ahead of print, 2020 Jun 9]. J Allergy Clin Immunol . 2020; S0091-6749(20)30828-9. doi:10.1016/j.jaci.2020.05.051

5. Avdeev S, Moiseev S, et al. Low prevalence of bronchial asthma and chronic obstructive lung disease among intensive care unit patients with COVID-19, Allergy. 2020. Doi: 10.1111/ALL.14420

6. Garg S, Kim L, Whitaker M, et al. Hospitalization Rates and Characteristics of Patients Hospitalized with Laboratory-Confirmed Coronavirus Disease 2019 - COVID-NET, 14 States, March 1-30, 2020. MMWR Morb Mortal Wkly Rep 2020;69:458-464. DOI: 10.15585/mmwr.mm6915e3

7. Gémes, K., Talbäck, M., Modig, K. et al. Burden and prevalence of prognostic factors for severe COVIDSweden. Eur J Epidemiol35, 401-409 (2020).https://doi.org/10.1007/s10654-020-00646-z

8. Williamson E, Walker AJ, Bhaskaran K, Bacon S, Bates C, Morton CE; The OpenSAFELY Collaborative. OpenSAFELY: factors associated with COVID-19-related hospital death in the linked electronic health records of 17 million adult NHS patients. 2020 May 7. medRxiv preprint doi:https://doi.org/10.1101/2020.05.06.20092999 